\title{
Einige notwendige Optimalitätsbedingungen für einfache reguläre Aufgabeı der optimalen Steuerung
}

\author{
Hoàng̉ Xû̃x Phú
}

Es werden drei notwendige Optimalitätsbedingungen für einfache reguläre Aufgaben der optimalen Steuerung mit Steuer- und Zustandsbeschränkungen bewiesẹn und dann zụ Lösung einer Aufgabenḳlasse angewandt.

Доказываются три необходимые условия оптшмальности для простых, регулярных задач оптимального управления с ограичениями управления и состояиия. Затем эти условия применяются для решенния некоторого класса задач.

Three necessily conditions for optimal solutions of special regular optimal control problenis with state and control constraints are proved. These conditions are then applied to a class of problems.

\section{Einleitung}

Wir betrachten die Aufgabe der optimalen Steuerung

$$
\begin{aligned}
& \therefore \mathfrak{F}(x, u):=\int_{t_{0}}^{t_{1}} f(t, x(t), u(t)) d t \rightarrow \text { inf! } \\
& \dot{x}(t)=u(t), \quad|u(t)| \leqq \beta, \quad|x(t)| \leqq \alpha \text { für } t \in\left[t_{0}, t_{1}\right], \quad x\left(t_{0}\right)=x\left(t_{1}\right)=0,
\end{aligned}
$$

wobei $\alpha, \beta>0$ und die Funktion $f: \dot{\mathbf{R}} \times \mathbf{R} \times \mathbf{R} \rightarrow \mathbf{R}$ stetig differenzierbar ist. Ferner ist $f(t, \xi, \cdot)$ zweimal stetig differenzierbar mit

$$
f_{v v}(t,-\xi, v)>0 \text { für alle }(t, \xi, v) \in\left[\iota_{0}, t_{1}\right] \times[-\alpha, \alpha] \times[-\beta, \beta],
$$

so daß (1)-(2) eine reguläre Aufgabe der optimalen Steuerung im Sinne eines Variationsproblems mit Steuer- und Zustandsbeschränkungen ist.

In weiteren wird die Funktion

$$
h(t, \xi, v, \dot{w}):=f_{\xi}(t, \xi, \dot{v})-f_{\iota t}(t, \xi, v)-f_{v \xi}(t, \dot{\xi}, \dot{v}) v-f_{v v}(t, \xi, v) w
$$

gebraucht. In ihr wird später anstelle von $w$ die Ableitung $\dot{u}$ der, Steuerung $u$ gesetzt. Das ist nur möglich, nachdem das Folgende gezeigt wurde.

Lem ma : Es sei $(x, u)$ ein optimaler Prozeß der Aufgabe (1)-(2). Dann ist u stetig und fast überall differenzierbar.

W.ir wollen hier den Beweis nicht vorfuihren, vgl. dazu [3, 5]. Ein klassisches Vor- : bild hierfür finden wir nach Weierstrass bei O. Bolza [1 : Seite 397]. 


\section{Einige notwendige Optimalitätsbedingungén}

Zuerst wollen wir eine notwendige Optimalitätsbedingung aus dem Pontrjaginschen Maximumprinzip ableiten.

Satz 1: Es sei $(x, u)$ ein optimaler Prozeß der Aufgube (1)-(2). Dann gilt

a) $h(t, x(t), u(t), \dot{u}(t))=0$ fast überall in $J_{0}(x, u)$,

b) $h(t, x(t), u(t), \dot{u}(t)) \leqq 0$ fust überall in $J_{1}(x, u)$,

c) $h(t, x(t), u(t), \dot{u}(t)) \geqq 0$ fast überall in $J_{2}(x, u)$ mil

$$
\begin{aligned}
& J_{0}(x, u): \doteq\left\{t \in\left[t_{0}, t_{1}\right]|| x(t)|<\alpha,| u(t) \mid<\beta\right\}, \\
& J_{1}(x, u):=\left\{t \in\left[t_{0}, t_{1}\right]|x(t)=\alpha,| u(t) \mid<\beta\right\}, \\
& J_{2}(x, u):=\left\{t \in\left[t_{0}, t_{1}\right]|x(t)=-\alpha,| u(t) \mid<\beta\right\} .
\end{aligned}
$$

Beweis: Nach dem Pontrjaginschen Maxinumprinzip (vgl. A. D. loffe und V. M. Trchomrrov [5: Seite 208] gilt für jeden optimalen Prozeß $(x, u)$ der Aufgabe $(1)-(2)$

$$
\begin{aligned}
& p(t)=l_{0}+\int_{t_{0}}^{t} \lambda_{0} f_{\xi}(t, x(t), u(t)) d t+\int_{\left[t_{0} . t\right)} d \mu_{1}-\int_{\left.\mid t_{0}, t\right)} d \mu_{2} ; \\
& p(t) u(t)-\lambda_{0} f(t, x(t), u(t))=\operatorname{Max}_{|v| \leqq \beta}[p(t) v-f(t, x(t), v)]
\end{aligned}
$$

fast überall in $\left[t_{0}, t_{1}\right]$, wobei $\lambda_{0} \geqq 0, \mu_{1}$ und $\mu_{2}$ nichtnegative, reguläre und auf $\left\{t \in\left[t_{0}, t_{1}\right] \mid x(t)=\alpha\right\}$ bzw. $\left\{t \in\left[t_{0}, t_{1}\right] \mid x(t)=-\alpha\right\}$ konzentrierte Maße sind. Man kann $\lambda_{0}>0$ zeigen. (vgl. [2]), deshalb kann $\lambda_{0}=1$ angenonmen werden. Daraus folgt wegen (ii)

$$
p(t)-f_{v}(t, x(t), u(t))=0 \quad \text { f. ï. in } J(x, u):=\left\{t \in\left[t_{0}, t_{1}\right]|| u(t) \mid<\beta\right\},
$$

weil $f$ in $v$ konvex ist. Durch Einsetzen in (i) ergibt sich wegen (3)

$$
\begin{aligned}
& h(t, x(t), u(t), \dot{u}(t)) \\
& =f_{\xi}(t, x(t), u(t))-\frac{d}{d t} f_{v}(t, x(t), u(t))=\frac{d}{d t} \cdot\left[\int_{\left(t_{0}, t\right)} d \mu_{1}-\int_{\left(t_{0}, t\right)} d \mu_{2}\right]
\end{aligned}
$$

fast überall in $J(x, u)$. Weil $\mu_{1}$ auf $\left\{t \in\left[t_{0}, t_{1}\right] \mid x(t)=\alpha\right\}$ und $\mu_{2}$ auf $\left\{t \in\left[t_{0}, t_{1}\right] \mid x(t)\right.$ $=-\alpha\}$ konzentriert und nicht negativ sind, ergibt sich die Behauptung des Satzes 1 unmittelbar aus dieser. Gleichung

Anhand der Funktion $h$ wird der Zustandsbereich

$$
G=\left[t_{0}, t_{1}\right] \times[-\alpha, \alpha]
$$

in drei Bereiche eingeteilt, die nicht unbedingt zusammenhängend sein müssen, nämlich

$$
\begin{aligned}
& G^{0}:=\{(t, \xi) \in G \mid h(t, \xi, 0,0)=0\}, \\
& G^{+}:=\{(t, \xi) \in G \mid h(t, \xi, 0,0)>0\}, \\
& G^{-}:=\{(t, \xi) \in G \mid h(t, \xi, 0,0)<0\} .
\end{aligned}
$$


Nun gilt

Satz 2: Es seï $(x, u)$ ein óptimaler Proze $\beta$ der Aufgabe (1)-(2), $G_{i}$ ein zusanmen: hïngender Bereich in $G$ und $z, s_{i-1}, s_{i} \in\left[t_{0}, t_{1}\right]$ mit $s_{i-1}<z<s_{i}$ derart, da $u(z)=0$ und $(t, x(t)) \in G_{i}$ für alle $t \in\left(s_{i-1}, s_{i}\right)$ gilt.

a). Im Falle $G_{i} \subset G^{+}$ist $u(t) \leqq 0$ für $t \in\left(s_{i-1}, z\right)$ und $u(t) \geqq 0$ für $t \in\left(z, s_{i}\right)$.'

b) Im Falle $G_{i} \subset G^{-}$ist $u(t) \geqq 0$ für $t \in\left(s_{i-1}, z\right)$ und $u(t) \leqq 0$ für $t \in\left(z, s_{i}\right)$.

Be-weis: Angenomimen, die Behauptung a) wäre falsch. Dann muß ein $z^{\prime} \in\left(s_{i-1} ; z\right)$ mit $u\left(z^{\prime}\right)>0$ oder ein $z^{\prime} \in\left(z, s_{i}\right)$ mit $u\left(z^{\prime}\right)<0$ existieren. Wir werdén nur den ersten Fall erörtern, für den zweiten Fall sind die Úberlegungen analog und b) kann analog zu a) bewièsen werden. Es sei also $u\left(z^{\prime}\right)>0$ für ein $z^{\prime} \in\left(s_{i-1}, z\right)$. Wegen der Stetigkeit von $u$ und $u(z)=0 \mathrm{mu}$ ein $z^{\prime \prime} \in\left(z^{\prime}, z\right]$ mit $u\left(z^{\prime \prime}\right)=0$ und $u(t)>0$ für alle $t \in\left[z^{\prime}, z^{\prime \prime}\right)$ existieren. Weil $u$ fast iiberall differenzierbar ist (vgl. Lemma 1), gilt für alle $\delta>0, \mathrm{da} B \dot{u}(t)<0$ für alle $t$ einer Menge von positivem $\mathrm{Ma} B$ in $\left(z^{\prime \prime}-\delta, z^{\prime \prime}\right)$ ist. Aus

$$
h(t, x(t), u(t), \dot{u}(t))-h(t, x(t), \dot{u}(t), 0)=-\dot{f}_{v v}(t, x(t), u(t)) \dot{u}(t)
$$

und (2) folgt weiter $h(t, x(t), u(t), \dot{u}(t))>h(t, x(t), u(t), 0)$ für alle $t$ einer Menge vom positiven $\mathrm{Ma} B$ in $\left(z^{\prime \prime}-\delta, z^{\prime \prime}\right)$. Da $x, u$ und $h$ stetig $\cdot \operatorname{sind}, h(t, x(t), 0,0)^{\prime}>0$ für $t \in\left[z^{\prime}, z^{\prime \prime}\right] \subset\left(s_{i-1}, s_{i}\right)$ und $u\left(z^{\prime \prime}\right)=0$ ist, läßt sich $\delta$ so klein wählen, da B gilt

$$
h(t, x(t), \dot{u}(t), 0)>0, \quad 0<u(t)<\beta \quad \text { und } \dot{z}^{\prime \prime}-\delta>z^{\prime}
$$

für alle $t \in\left(z^{\prime \prime}-\delta, z^{\prime \prime}\right)$. Für dieses $\delta$ gilt also $h(t, x(t), u(t), \dot{u}(t))>0$ für alle $t$ einer Menge von positivem Ma 3 in $\left(z^{\prime \prime}-\delta, z^{\prime \prime}\right)$, im Widerspruch zu Satz 1 , denn wegen $0<u(t)<\beta$ und $\dot{x}(t)=u(t)$ für $\iota$ aus $\left(z^{\prime \prime}-\delta, z^{\prime \prime}\right)$ gilt $\left(z^{\prime \prime}-\delta, z^{\prime \prime}\right) \subset J_{0}(x, u)$

Folgerung 1 : Es mögen $(x, u), G_{i}$ und $s_{i-1}$, $s_{i}$ den Voraussetzungen in Satz 2 genügen. Dann gelten jolgende. Aussagén:

a) Im Falle $G_{i} \subset G^{+}, z \in\left(s_{i-1}, s_{i}\right)$ und $u(z)>0$ ist $u(\iota)>0$ für alle $t \in\left(z, s_{i}\right)$.

b) Im Falle $G_{i} \subset G^{-}, z \in \cdot\left(s_{i-1}, s_{i}\right)$ und $u(z)<0$ ist $u(t)<0$ fürr alle $t \in\left(z, s_{i}\right)$.

Beweis: Angenomimen, die Béhauptung a) wäre falsch. Dann existiert wegen dẹ Stetigkeit von $u$ ein $z^{\prime} \epsilon \cdot\left(z, s_{i}\right)$ mit $u\left(z^{\prime}\right):=0$. Nach Sat 2 muß $u(z) \leqq 0$ sein, weil $z \in\left(s_{i-1}, z^{\prime}\right)$ ist, in Widerspruch zur Voraussetzung. Der Beweis für b) verläuft analog

Definition: Es heißt $z \in\left(t_{0}, t_{1}\right)$ lokal sirenge Maximalstelle (bzw. Minimalstelle) der Funktion $x$, wenn für ein $\delta_{1}>0$ gilt

$$
|t-z|<\delta_{1} \quad \text { impliziert } \quad x(\ell) \leqq x(z), \quad \text { (bzw. } x(t) \geqq x(z) \text { ) }
$$

und wenn fiir alle $\delta_{2}>0$ ein $z^{\prime}$ existiert mit

$$
\left|z-z^{\prime}\right|<\delta_{2} \quad \text { und } x\left(z^{\prime}\right)<x(z) \quad\left(\text { bzw. } x\left(z^{\prime}\right)>x(z)\right)
$$

Mit diesen Begriffen formulieren wir jetzt eine andere notwendige Optimalitätsbedingung, die sich später in den Anwendungen als sehr wirksam erweisen wird.

Satz 3: Es. sei $(x, u)$ ein optimaler Prozeß der Aufgabe (1)-(2). Dann besitzt $x$ keine lokal strenge Maximalstelle in $G^{+}$und keine lokal strenge Minimalstelle in $G^{-}$.

Beweis: Angenommen, $z$ wäre eine lokal strenge Maximalstelle von $x$ in $G^{+}$(der Beweis bétreffs, einer lokal strengen Minimalstelle in $G^{-}$verläuft analog). Wegen 
der Stetigkeit von $u$ und $\dot{x}=u$ gilt dann $u(z)=0$. Wegen der Definition von $G^{\dot{*}}$ existieren $s_{i-1}, s_{i} \in\left[t_{0}, t_{1}\right]$ und $G_{i} \subset G^{+}$mit

$$
z \in\left(s_{i-1}, s_{i}\right) \text { und }(t, x(t)) \in G_{i} \text { für alle } t \in\left(s_{i-1}, s_{i}\right) \text {. }
$$

Aus Sat $z 2$ folgt $u(t) \leqq 0$ fiir alle $t \in\left(\dot{s}_{i-1} ; z\right)$ und $u(t) \geqq 0$ fiir alle $t \in\left(z, s_{i}\right)$. Deshalb gilt $x(t) \geqq x(z)$ für alle $t \in\left(s_{i-1}, s_{i}\right)$, im Widerspruch zur Definition der lokal strengen Maximalstelle

\section{Anwendungsbeispiele}

Mit Hilfe der im Abschnitt 1 bewiesenen Optimalitätsbedingungen kann man schon viele Aufgaben der optimalen Steuerung lösen. Hier wollen wir einige ty pische Beispiele bëtrachten.

Beispiel 1: Wir betrachten die Aufgabe

$$
\begin{aligned}
& \int_{0}^{T}\left(u^{2}-u x-\cos x\right) d t \rightarrow \inf ! \\
& \dot{x}=u, \quad|u| \leqq \beta, \quad|x| \leqq \alpha, \quad, \quad x(0)=x(T)=0 .
\end{aligned}
$$

$(0<\alpha<\pi)$. Für diese ist $h(t, \xi, v, w)^{\circ}=\sin \xi-2 u$. Der Zustandsbereich $G=[0, T]$ $\times[-\alpha, \alpha]$ wird anhand $h(\cdot, \cdot, 0,0)$ in die drei Bereiche

$$
\begin{aligned}
& G_{1}:=\{(t, \xi) \in G \mid 0<\xi \leqq \alpha\}=G^{+}, \\
& G_{2}:=\{(t, \xi) \in G \mid \xi==0\}=G^{0}, \\
& G_{3}:=\{(t, \xi) \in G \mid 0>\xi \geqq-\alpha\}=G^{-}
\end{aligned}
$$

eingeteilt. (vgl. Abb.,1).

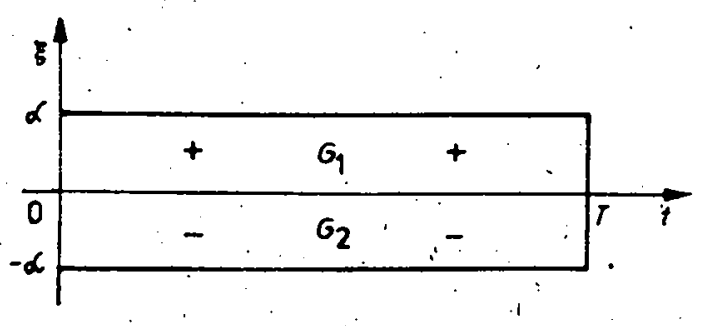

Abb. 1

Es sei $(x, u)$ ein optimaler Prozeß dieser Aufgabe. Dän kann $x$ nicht in $G_{1}$ eintreten; denn sonst existiert wegen $x(0)=x\left(T^{\prime}\right)=0$ mindestens eine lokal strenge Maximalstelle in $G_{1}=G^{+}$, im Widerspruch zu Satz 3. Ebenso kann $x$ nicht in $G_{3}$ eintreten; - sonst existiert eine lokal strenge Minimalstelle in $G_{3}=G^{-}$. Also-kann $x$ nur noch in $G_{2}$ bleiben. Ausgehend von der Existenz des optimalen Prozesses (vgl. [5, 7]) bedeutet das: Der einzige optimale Proze $B$ dieser Aufgabe ist $(x, u)$ mit $x(t)=0$, $u(t)=0$ für alle $t \in[0, T]$.

Beispiel 2: Wir betrachten die Aufgabe

$$
\begin{aligned}
& \int_{0}^{T}\left(u^{2}-\sin x+x^{2}\right) d t \rightarrow \inf ! \\
& \dot{x}=u, \quad-|u| \leqq \beta, \quad|x| \leqq \alpha, \quad x(0)=x(T)=\dot{0} .
\end{aligned}
$$


$(1 / 2<\alpha<x / 2, T$ und $\beta$ hinreichend groß). Für diese ist $h(t, \xi, v, w)=-\cos \xi$ $+2 \xi-2 w$. Es sei $\xi_{0}$ die Lösung der Gleichung $-\cos \xi+2 \xi=0$. ( $\xi_{0}$ ist eindeutig und annähernd glëich 0,45$)$. Dann wird der Zustandsbereich $G$ anhand $h(\cdot, \cdot, 0,0)$ in die fuinf Bereiche

$$
\begin{array}{ll}
G_{1}:=\left\{(t, \xi) \in G \mid \xi>\xi_{0}\right\}=G^{+}, & G_{4}:=\{(t, \xi) \in G \mid \xi=0\} \subset G^{-}, \\
G_{2}:=\left\{(t, \xi) \in G \mid \xi=\xi_{0}\right\}=G^{0}, & G_{5}:=\{(t, \xi) \in G \mid \xi<0\} \subset G^{-} \\
G_{3}:=\left\{(l, \xi) \in G \mid 0<\xi<\xi_{0}\right\} \subset G^{-}, &
\end{array}
$$

eingeteilt (vgl. Abb. 2).

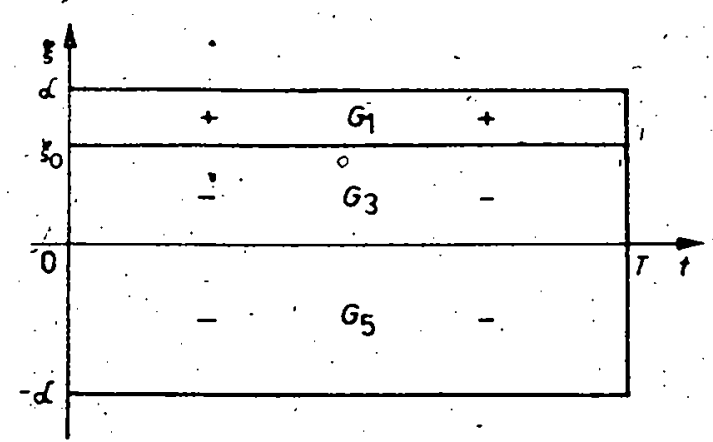

Abb. 2

Wenn wir diese Aufgabe mit dem in [5] dargestellten Pontrjaginschen Maximumprinzip lösen, dann kommen wegen der verschiedenén möglichen 'Konstanten und Maße zunächst alle $G_{i}$ in Betracht. Das ist natïrlich sehr umfangreich. Mit Hilfe der Sätze"1-3 kann aber die Untersuchung erheblich verkiirzt: werden: Analog wie in' Beispiel 1 kann kein optimaler Proze $\beta$ in $G_{1}=G^{+}$eintreten, sonst.würde eine lokal'strenge Maximalstelle von $x$ in $G^{+}$existieren, im. Widerspruch zu Satz 3. Ebenso kann er auch nicht in $G_{5}$ eintreten, sonst gäbe es eine lokal strenge Minimalstelle von $x$ in, $G^{-}$. Der optimale Proze $B(x, u)$ kann ebenfalls nicht in $G_{4}$ bleiben, d. h. $x(t)=0, ; u(t)=0$ für alle $t \in[0, T]$ sein, denn sonst wäre $h(t, x(t), u(t), \dot{u}(t))$ $=h(t, 0,0,0)<0$ fiir alle $t \in[0, T]=J_{0}(x, u)$, im Widerspruch zù Satz 1. Also mu $\beta$. die Untersuchung nur noch in $G_{2} \cup G_{3} \cup\{(0,0),-(0, T)\}$ durchgefiihrt werden (vgl. Abb. 3). Nun betrachten wir den Prozeß $\left(x^{*}, u^{*}\right)$ mit

$$
\begin{aligned}
& x^{*}(0)=x^{*}\left(T^{\prime}\right)=0, \quad z_{1}<z_{2}, \\
& x^{*}(t)=\xi_{0}, \quad \iota^{*}(t)=0 \text { für alle } t \in\left[z_{1}, z_{2}\right], \\
& 2 x^{*}(t)-\cos x^{*}(t)-2 \ddot{x}^{*}(t)=0 \text { für alle } t \in\left(0, z_{1}\right) \cup\left(z_{2}, T\right)
\end{aligned}
$$

( $T$ ' und $\beta$ seien so gro $\beta$, da $\beta z_{1}$ und $z_{2}$ mit den obigen Eigenschaften existieren und $\beta>u^{*}(0), \beta>-u^{*}\left(T^{\prime}\right)$ gilt). Es läßt sich zeigen, da $\beta\left(x^{*}, u^{*}\right)$ sowie $z_{1}$ und $z_{2}$ dadurch eindeutig definiert sind. Man kann auch nachprüfen, daß dieser Proze $\beta$ der im Satz 1 angegebenen notwendigeñ Optimalitätsbedingung geniigt; d. h. daß $h\left(t, x^{*}(t), u^{*}(t), u^{*}(t)\right)=0$ fïr alle $\ell \in[0, T]=J_{0}\left(x^{*}, u^{*}\right)$ ist.

Wir wollen jetzt den Beweis dafiir skizzieren, da $\beta\left(x^{*}, u^{*}\right)$ der einzige optimale Proze $\beta$ ist. Es sei zuerst $(x, u)$ ein optimaler Proze $B$ mit $u(0)>u^{*}(0)$. Jann kann man zeigen, da $B$ in $G_{3}$ obeṛhalb von $x^{*}$ liegt. Ist $z_{3}$ dèrjenige Zeit punkt mit $x\left(z_{3}\right)=\xi_{0}$; dann gilt $u\left(z_{3}\right) \geqq u^{*}\left(z_{3}\right)>0$. Also tritt $x$ in $G_{1}$ ein, im Widerspruch zur obigen Úberlegung. Nun sei $(x, u)$ ein optimaler Prozeß mit $u(0)<u^{*}(0)$. Dañn kann man 


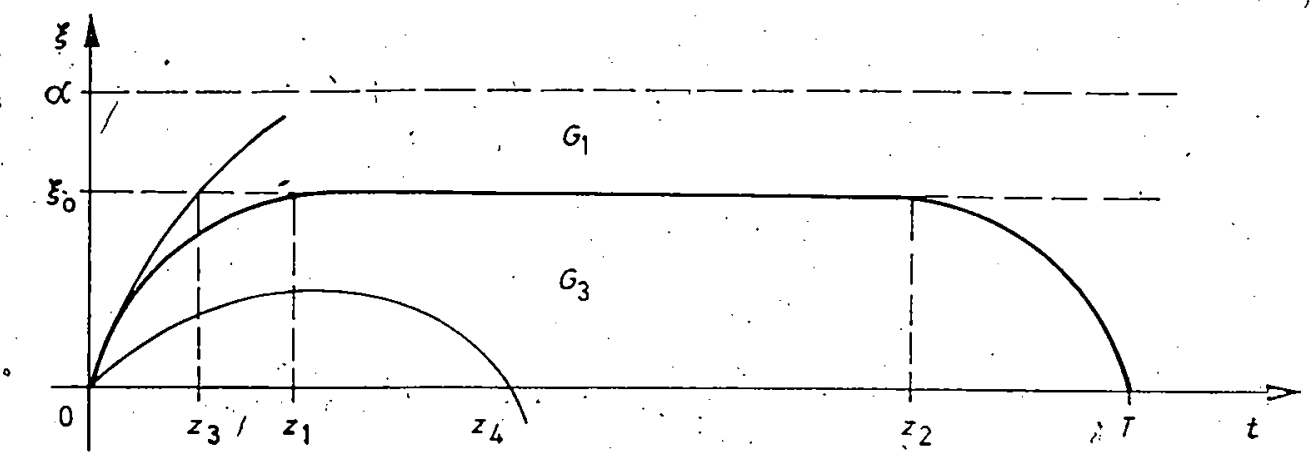

Abb. 3

zeigen, da $\beta x$ unterhalb von $x^{*}$ verläuft und die $t$-Achse in $z_{4}^{\circ}$ mit $z_{4}<T$ schneidet. Dabei gilt $u\left(z_{4}\right)<0$, d. h. $x$ tritt in $G_{5}$ ein, ebenfalls im Widerspruch zur obigen Überlegung. Damit muß $u(0)=u^{*}(0)$ für einen beliebigen optimalen Proze $\mathcal{B}(x, u)$ gelten. Wegen der Findeutigkeit der Lösung der zugehörigen Differentialgleichung stimmen $\left(x^{*}, u^{*}\right)$ und $(x, u)$ in $\left[0, z_{1}\right]$ überein. Analog kann man die Úbereinstimmung $(x, u)$ und $\left(x^{*}, u^{*}\right)$ in $\left[z_{2}, T\right]$ beweisen. In $\left(z_{1}, z_{2}\right)$ schließlich müssen $(x, u)$ und $\left(x^{*}, u^{*}\right)$ auch identisch sein, denn sonst verliefe $x$ innerhalb von $G_{3}$, und wegen $x\left(z_{1}\right)=x\left(z_{2}\right)=\xi_{0}$ gäbe es mindestens eine lokal strenge Minimalstelle in $G_{3} \subset G^{-}$, im Widerspruch zu Satz 3. Ausgehend von der Existenz eines optimalen Prozesses (vgl. [5, 7]) folgt daraus, da $\beta\left(x^{*}, u^{*}\right)$ der einzige'optimale Proze $B$ ist.

Beispiel 3: Wir betrachten die Aufgabe

$$
\begin{aligned}
& \int_{0}^{T}\left((t+1) u^{2}-u x+\sin x\right) d t \rightarrow \text { inf } ! \\
& \dot{x}=u, \quad \cdot \quad|u| \leqq \beta, \quad|x| \leqq \alpha, \quad x(0)=x(T)=0
\end{aligned}
$$

$(0<\alpha<\pi / 2, T$ und $\beta$ hinreichend groß). Fiur diese ist $h(t, \xi, v, w)=\cos \xi-2 v$ $-2(t+1) w$. Damit ist $h(t, \xi, 0,0)>0$ für alle $(t, \xi) \in G$, d. h. $G^{+}=G$ (vgl. Abb. 4). Analog wie im Beispiel 2 kann man zeigen, daß der wie folgt definierte Prozeß $\left(x^{*}, u^{*}\right)$ der einzige optimale Prozeß dieser Aufgabe ist. (vgl. Abb. 5):

$$
\begin{aligned}
& x^{*}(0)=x^{*}(T)=0, \quad z_{1}<z_{2}, \\
& x^{*}(t)=-\alpha, \quad u^{*}(t)=0 \text { fiir } t \in\left[z_{1}, z_{2}\right] \\
& \cos x^{*}(t)-2 x^{*}(t)-2(t+1) \ddot{x}^{*}(t)=0 \text { fiir } t \in\left(0, z_{1}\right) \cup\left(z_{2}, T\right) .
\end{aligned}
$$

Beispiel 4: Wir betrachten die Aufgabe

$$
\begin{aligned}
& \mathfrak{F}(x, u)=\int_{0}^{r}\left(u^{2}-\sin ^{2} x+\frac{x^{2}}{2}\right) d t \rightarrow \text { inf } ! \\
& \dot{x}=u, \quad|u| \leqq \beta, \quad|x| \leqq \alpha, \quad x(0)=x(T)=0
\end{aligned}
$$

$(0<\alpha \leqq \pi / 4, T$ und $\beta$ hinreichend groß). Dies ist die schwierigste unter den vier behandelten Beispielen. Sie wurde in [2] aușfïhrlich behandelt. Hier wird die Lösung 


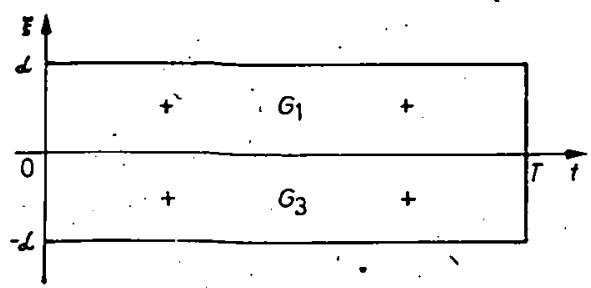

Abb. 4

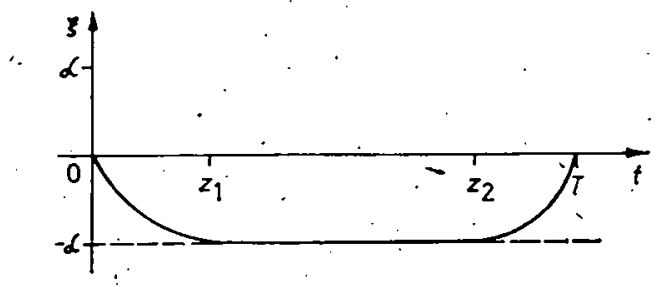

Abb. 5

nur angegeben. Es ist

$$
h(t, \xi, v, w)=-\sin 2 \xi+\xi-2 w .
$$

Der Zustandsbereich wird in die drei Bereiche.

$$
\begin{aligned}
& G_{1}:=\{(t, \xi) \in G \mid \xi>0\}=G^{-} \\
& G_{2}:=\{(t, \xi) \in G \mid \xi=0\}=G^{0} \\
& G_{3}:=\{(t, \xi) \in G \mid \xi<0\}=G^{+}
\end{aligned}
$$

zerlegt (vgl. Abb. 6). Wir betrachten drei Prozesse $\left(x_{1}, u_{1}\right),\left(x_{2}, u_{2}\right)$ und $\left(x_{3}, u_{3}\right)$ mit

$$
\begin{aligned}
& x_{1}(0)=x_{1}(T)=0, \quad z_{1}<z_{2}, \\
& x_{1}(t)=\alpha, \quad u_{1}(t)=\dot{0} \quad \text { für alle } t \in\left[z_{1}, z_{2}\right], \quad \\
& -\sin 2 x_{1}(t)+x_{1}(t)-2 \ddot{x}_{1}(t)=0 \text { für alle } t \in\left(0, z_{1}\right) \cup\left(z_{2}, T\right) \\
& x_{3}(t)=-x_{1}(t) \quad \text { und } x_{2}(t)=0 \text { für alle } t \in[0, T]
\end{aligned}
$$

(vgl. Abb. 7). Diese genügen der im Satz 1 dargestellten notwendigen Optimalitätsbedingung. Jedoch kommen nur $\left(x_{1}, u_{1}\right)$ und $\left(x_{3}, u_{3}\right)$ in Frage, weil $\mathfrak{F}\left(x_{1}, u_{1}\right)=\mathfrak{F}\left(x_{3}, u_{3}\right)$ $<0=\mathfrak{F}\left(x_{2}, u_{2}\right)$ gilt. Ferner kann man zeigen, daß es keinen optimalen Prozeß gibt, der von $\left(x_{1}, u_{1}\right)$ und $\left(x_{3}, u_{3}\right)$ verschieden ist. Ausgehend von der Existenz der optimalen Prozesse müssen $\left(x_{1}, u_{1}\right)$ und $\left(x_{3}, u_{3}\right)$ die einzigen optimalen Prozesse dieser Aufgabe sein.

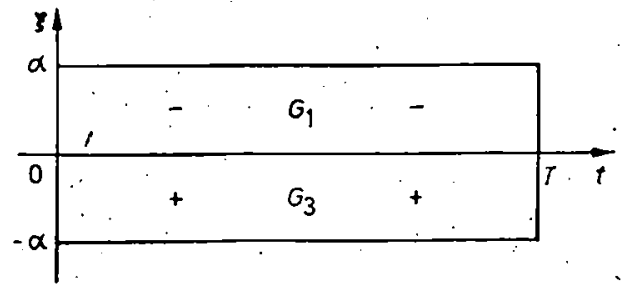

Abb. 6

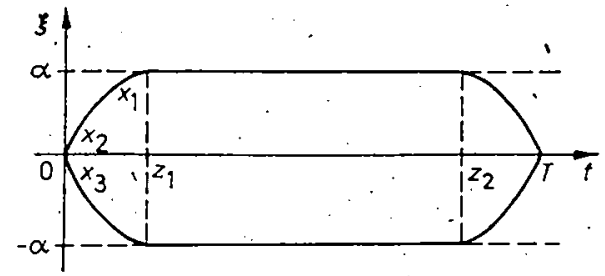

Abb. 7

Damit haben wir gesehen, wie die vorangestellten notwendigen Optimalitätsbedingungen die Konstruktion optimaler 'Trajektorien wesentlich vereinfachen können. Dièses Konstruktionsprinzip nennen wir Methode der Bereichsanalyse. Sie wird in anderen Fällen auch in [4] beschrieben. 
Wir haben vier Beispiele untersucht. Diese besitzen die gemeinsame Eigenschaft

$$
\begin{aligned}
& \frac{\partial}{\partial t}\left(f_{\xi}(t, \xi, v)-f_{v t}(t, \xi, v)\right)=0 \text { für alle }(t, \xi, v) \in\left[t_{0}, t_{1}\right] \times[-\alpha, \alpha] \\
& \bar{x}[-\beta, \beta] .
\end{aligned}
$$

Unter dieser Voraussetzung kann die Aufgabe (1)-(2) für hinreichend kleines $\alpha$ außer den in den Beispielen 1-4 auftretenden Typen der Struktur von $G$ nur noch die zwei in Abb. 8 und 9 dargestellten Typen haben, die sich aber ähnlich wie in den Beispielen 2 und 3 behandeln lassen. Dabei sind die mit ,+" und ,- " markierten Bereiche.in $G^{+}$bzw. $G^{-}$enthalten.

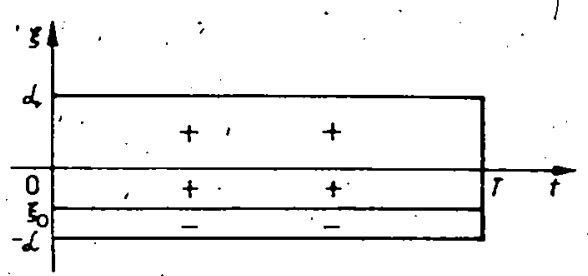

Abb. 8

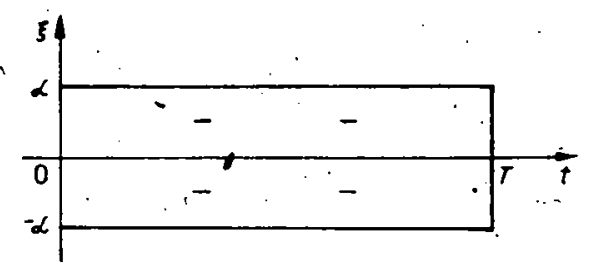

Abb. 9

Schließlich wollen wir noch ein angewandtes Beispiel untersuchen.

Beispiel 5: Gegeben sei cine Kurve $K(\cdot):[0, T] \rightarrow \mathbf{R}$ und eine Zahl $\alpha>0$ nit $K(t)>\alpha$ fiur $t \in[0, T], K(\cdot) \in C^{2}[0, T]$. Gesucht ist eine Kurve $y(\cdot):\left[0,{ }^{\prime} T\right] \rightarrow \mathbf{R}$ mit

$$
y(0)=K(0), \quad y(T)=K(T) \quad \text { und } \quad|y(t)-K(t)| \leqq \alpha \quad \text { für } t \in[0, T] \text {, }
$$

so daß die Rotationsfläche, die durch Drehung des Kurvenbogens $y(\cdot)$ un die tAchse entsteht, einen minimalen Inhalt

$$
J(y)^{\prime}=2 \pi \int_{0}^{T} y \sqrt{1+\dot{y}^{2}} d t
$$

ihrer Mantelfläche' besitzt. Un diese Aufgabe in die Gestalt von (1) umzuformen, fiihren wir $x:=y-K$ als neue Variable ein. Damit entsteht die äquivalente Aufgabe

$$
\begin{aligned}
& \Im(x, u):=\int_{0}^{T}(x+K(t)) \sqrt{1+(u+\dot{K}(t))^{2}} d t \rightarrow \text { inf ! } \\
& \dot{x}=u, \quad|u| \leqq \beta, \quad|x| \leqq \alpha ; \quad x(0)=x(T)=0 .
\end{aligned}
$$

In folgenden untersuchen wir diese Aufgabe für hinreichend kleines $\alpha$ und hinreichend großes $\beta$.

Die Funktion $h$ lautet

$$
\begin{aligned}
h(t, \xi, v, w)= & {\left[1+(v+\dot{K}(t))^{2}\right]^{-3 / 2}\left[1+(v+\dot{K}(t))^{2}-(\xi+K(t))\right.} \\
, & \times(w+\ddot{K}(t))]
\end{aligned}
$$

und damit ist

$$
h(t, \xi, 0,0)=\left[1+\dot{K}^{2}(t)\right]^{-\dot{3} / 2}\left[1+\dot{K}^{2}(t)-(\xi+K(t)) \ddot{K}(t)\right] .
$$


'Wir betrachten dazu zwei besondere Fälle:

Fall $a: 1+\dot{K}^{2}(t)-(\alpha+K(t)) \ddot{K}(t)>0$ für alle $t \in[0, T]$. (Zu beachten ist, da $\beta$ dabei $\ddot{K}(t)$ wegen $K(t)-\alpha>0$ auch negativ sein kann.). In diesem Falle gilt .

$$
1+\dot{K}^{2}(t)-(\xi+\dot{K}(t)) \ddot{K}(t)>0 \text { für } t \in[0, T] \text { und } \xi \in[-\alpha, \alpha] \text {, }
$$

d. h. $G=G^{+}$. Analog wie im Beispiel 3 gilt nun, daß der wie folgt definierte Prozeß $\left(x^{*}, u^{*}\right)$ optimal ist:

$$
\begin{aligned}
& x^{*}(0)=x^{*}\left(I^{\prime}\right)^{\prime}=0, \quad z_{1}<z_{2}, \\
& x^{*}(t)=-\alpha, \quad u^{*}(t)=0 \text { für } t \in\left[z_{1}, z_{2}\right], \\
& h\left(t, x^{*}(t), u^{*}(t), \dot{u}^{*}(t)\right)=0 \text { für } t \in\left(0, z_{1}\right) \cup\left(z_{2}, J^{\prime}\right):
\end{aligned}
$$

(Die Existenz und die Findeutigkeit von $\left(x^{*}, u^{*}\right)$ ist für hinreichend kleines $\alpha$ gewährleistet.) Im Innern des Zustandsbereiches hat $x^{*}$ die Gestalt

$$
x^{*}(t)=c_{1}^{-1} \cosh \left(c_{1} t+c_{2}\right)-K(t)
$$

wobei $c_{1}$ und $c_{2}$ jeweils in $\left(0, z_{1}\right)$ bzw. $\left(z_{2}, T\right)$ geeignete Konstanten sind (vgl. 'A. KNeschke [6: Seite 287]). Wenn wir das Ergebnis auf-die ursprüngliche Form zuriickfuihren, hat die optimale Kurve $y^{*}(\cdot)$ in diesem Fall die Gestalt

wobei

$$
\begin{aligned}
& y^{*}(t)=K(t)-a \text { für } t \in\left[z_{1}, z_{2}\right] \\
& y^{*}(\dot{t})=c_{1 i}^{-1} \cosh \left(c_{1 i} t+c_{2 i}\right) \text { fïr } t \in I_{i}
\end{aligned}
$$

$$
\begin{aligned}
& I_{1}=\left[0, z_{1}\right], \quad c_{11}=\frac{\sqrt{1+\dot{K}^{2}\left(z_{1}\right)}}{K\left(z_{1}\right)-\alpha}, \quad c_{21}=\operatorname{arcosh}\left(c_{11} K(0)\right) \\
& I_{2}=\left[z_{2}, T\right], \quad c_{12}=\frac{\sqrt{1+\dot{K}^{2}\left(z_{2}\right)}}{K\left(z_{2}\right)-\alpha}, \quad c_{22}=\operatorname{arcosh}\left(c_{12} K(T)\right)-c_{12} T
\end{aligned}
$$

ist (vgl. dazu Abb. 10).

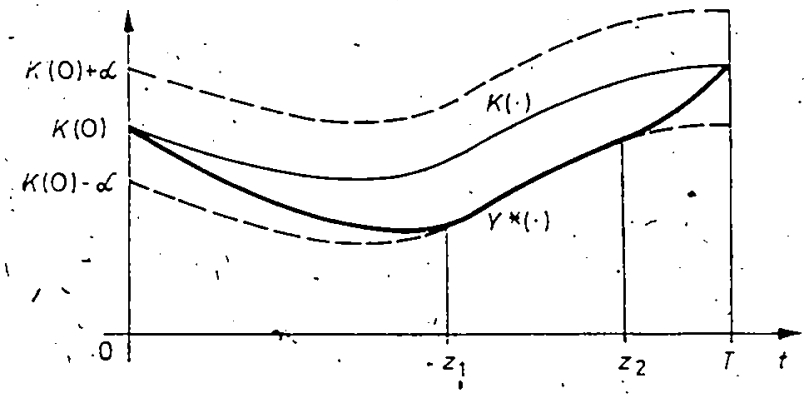

Abb. 10 .

Fall $b: 1+\dot{K}^{2}(t)+(\alpha-K(t)) \ddot{K}(t)<0$ für alle $t \in[0, T]$. In diesem Falle iśt $G=G^{-}$. Für hinreichend kleines $\alpha$ existiert genau ein Prozeß $\left(x^{*}, u^{*}\right)$ mit.

$$
\begin{aligned}
& x^{*}(0)=x^{*}\left(T^{\prime}\right)=0, \quad z_{1}<z_{2}, \\
& x^{*}(t)=\alpha, \quad u^{*}(t)=0 \text { für } t \in\left[z_{1}, z_{2}\right], \ldots \\
& h\left(t, x^{*}(t), u^{*}(t) ; \dot{u}^{*}(t)\right)=0 \text { für } \iota_{1} \in\left(0, z_{1}\right) \cup\left(z_{2}, T\right)
\end{aligned}
$$




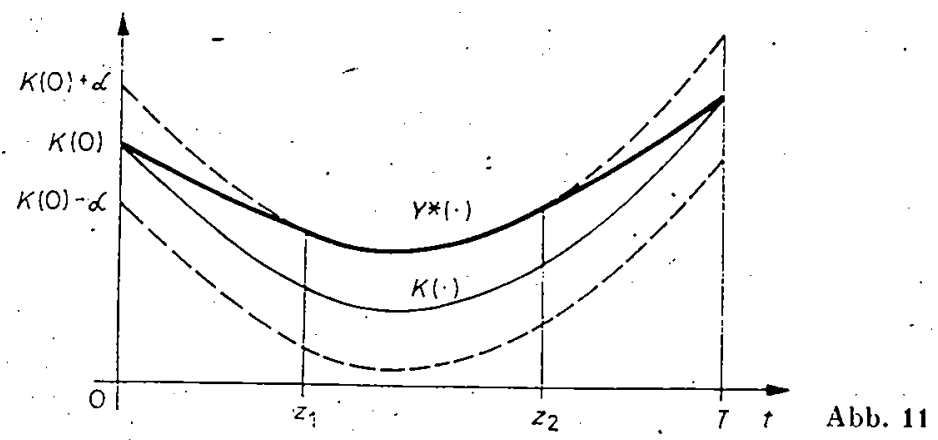

Analog wie oben kann man zeigen, da $\beta^{*}\left(x^{*}, u^{*}\right)$ der einzige optimale Prozeß ist. Weil es sich aus der letzten Gleichung ergibt, da $B$ im Innern des Zustandsbereiches $x^{*}$ die Gestalt

$$
x^{*}(t)=c_{1}^{-i} \cosh \left(c_{1}^{\prime} t+c_{2}\right)-K(t)
$$

hat, kann man zeigen, da $y^{*}(\cdot)$ mit

$$
\begin{aligned}
& y^{*}(t)=K(t)+\alpha \text { für } t \in\left[z_{1} ; z_{2}\right], \\
& y^{*}(t)=c_{1 i}^{-1} \cosh \left(c_{1 i} t+c_{2 i}\right) \text { fïr } t \in I_{i}
\end{aligned}
$$

die einzige optimale Kurve der ursprünglichen Aufgabe ist, wobei

$$
\begin{aligned}
& I_{1}=\left[0, z_{1}\right], \quad c_{11}=\frac{\sqrt{1+\dot{K}^{2}\left(z_{1}\right)}}{K\left(z_{1}\right)+\alpha}, \quad c_{21}=\operatorname{arcosh}\left(c_{11} K(0)\right) \\
& I_{2}=\left[z_{2}, T\right], \quad c_{12}=\frac{\sqrt{1+\dot{K}^{2}\left(z_{2}\right)}}{K\left(z_{2}\right)+\alpha}, \quad c_{22}=\operatorname{arcosh}\left(c_{12} K(T)\right)-c_{12} T
\end{aligned}
$$

ist (vgl. dazu Abb. 11).

Das Beispiel 5 ist verwandt mit folgender praktischer Aufgabe: Gesucht ist die stabile Lage $y(\cdot)$ cines Seils, das in einer Röhre liegt und dessen Enden an zwei gegebenen Punkten festgebunden sind. Das ist gerade die Lage, in der die potentielle Energie

$$
\varrho g \int_{0}^{T} y \cdot \sqrt{1+\dot{y}^{2}} d t
$$

minimalen Wert hat ( $a$ : Massendichte des Seils, $g$ : Erdbeschleunigung). Gemeinsam mit der Längerestriktion des Seils

$$
\int_{0}^{T} \sqrt{1+\dot{y}^{2}} d t=l
$$

hat diese Aufgabe die Form des Lagrange-Problems

$$
\int_{0}^{T}(y+\lambda) \sqrt{1+\dot{y}^{2}} d t \rightarrow \operatorname{Min} !
$$

Unter Anwendung der obigen Lösungsmethode und der Bestimniung des Zusammenhangs $z$ wischen dem Lagrange-Multiplikator $\lambda$ und der Länge $l$ kann man schlie $B$ lich analog die stabile Lage des Seils mit einer vorgegebenen Länge $t$ ermitteln. 


\section{LITERATUR}

[1] BoLza, O.: Vorlesungen über Variationsrechnung: Leipzig: BSB B. G. Teubner Verlags. gesellschaft 1909.

[2] Hod̀ng Xồ Prú: Methoden zur Lösung von Aufgaben der optimalen Steuerung mit engen Zustandsbereichen. Dissertation. Leipzig: Karl-Marx.Universität 1983.

[3] Hoàxg Xồ Puú:. Zur Stetigkeit der Lösung der adjungierten Gleichung bei Aufgaben der optimalen Steuerứg mit Zustandsbeschränkungen. Z. Anal. Anw. 6 (1984), $527-539$.

[4] Hod̀xg XUÂx PuÚ: Lösung einer einfachen regulären Aufgabe der optimalen Steuerung mit engen Zustandsbereichen anhand der Methode der Bereichsanalyse. Math. Operations. forsch. Statist., Ser. Optimization 16 (1985), $431-438$.

[5] Ioffe, A. D., und V. M. Tichomirov: Theorie der Extremalaufgaben. Berlin: Dt. Verlag IViss. 1979.

[6] KNeschke, A.: Differentialgleichungen und Randwertprobleme, Bd. ILI. Leipzig: BSB B. G. Teubner Verlagsgesellschaft 1962.

[7] Морьухович, Б. Ш.: Сущестнование оптимальных управленит̆: Итоги иауки, серия: Современные ироблемы математики 6. Москва 1976, 207.

Manuskripteingang: 25.06.1983; in revidierter Fassung 27.06. 1985

\section{VERFASSER:}

Dr. Hoàng Xuân Pró

Sektion Mathematik der Karl-Marx-Universität.

DDR - 7010 Leipzig, Karl-Marx-Platz

- Ab April 1987: Viện Toán học

Institute of IIathematics

P.o. BOX 631 Bò H’ô

Hanoi, Vietnam 